\title{
Subacute-onset paraneoplastic autonomic neuropathy associated with prostate cancer
}

\author{
Lorenzo Muccioli $^{1} \cdot$ Marianna Nicodemo $^{2,3} \cdot$ Pietro Cortelli $^{1,3} \cdot$ Maria Guarino ${ }^{2,3}$ (1)
}

Received: 28 July 2020 / Accepted: 13 August 2020 / Published online: 20 August 2020

(c) Springer-Verlag GmbH Germany, part of Springer Nature 2020

\section{Dear Editors,}

Isolated autonomic failure of acute or subacute onset suggests an immune cause, such as autoimmune autonomic ganglionopathy (AAG) and paraneoplastic autonomic neuropathy/ganglionopathy (PAN) [1]. While PAN may present with a limited enteric neuropathy, both disorders may present with diffuse autonomic failure and be clinically indistinguishable. Characteristic features include orthostatic hypotension, constipation and cholinergic failure such as sicca syndrome, urinary retention and impaired pupil responses. The antibodies most commonly associated with PAN might be directed to intracellular (anti-Hu, anti-CV2) or surface antigens (anti-ganglionic nicotinic acetylcholine receptor [anti-gAchR]) [1]. The former are onconeural antibodies strongly associated with underlying malignancy, most frequently small cell lung carcinoma (SCLC), and related to other paraneoplastic neurological syndromes [2,3]. Anti$\mathrm{gAchR}$ antibodies are identified at high levels $(>1.0 \mathrm{nmol} / \mathrm{L})$ in more than half of patients with AAG and, in a minority of cases (about 15\%), are associated with underlying malignancy (SCLC, thymoma or adenocarcinomas) [1, 4].

Here, we report the case of an elderly patient in whom subacute-onset pandysautonomia lead to the diagnosis of metastatic prostate cancer associated with low titer antigAchR antibodies.

A 75-year-old-man with a past history of benign prostate hyperplasia presented at the emergency department with acute urinary retention. A urethral catheter was placed,

Maria Guarino

maria.guarino@aosp.bo.it

1 Department of Biomedical and Neuromotor Sciences, University of Bologna, Bologna, Italy

2 Neurology Unit, S. Orsola-Malpighi University Hospital, Via Giuseppe Massarenti 9, 40139 Bologna, IT, Italy

3 IRCCS Istituto Delle Scienze Neurologiche Di Bologna, Bologna, Italy resulting in drainage of ca. $1000 \mathrm{cc}$ of urine, and the patient was dismissed with a urology appointment scheduled. After 2 days, he had several syncopal episodes while standing during the morning, and was therefore admitted for further investigation. He was found to have orthostatic hypotension, with a blood pressure drop 3 min after standing of $30 / 20 \mathrm{mmHg}$ (from 130/80 to $100 / 60 \mathrm{mmHg}$, heart rate of $70 \mathrm{bpm}$, unchanged). Moreover, because of rapidly progressive abdominal bloating and constipation, he underwent an abdominal radiography and, subsequently, colonoscopy, which were consistent with acute colonic pseudo-obstruction (ACPO) [Fig. 1]. Abdominal ultrasound also showed bilateral ureteronephrosis. Neurological examination was unremarkable and, specifically, no sensory-motor symptoms or signs were present.

Considering the isolated subacute-onset pandysautonomia of possible paraneoplastic origin, the patient underwent a contrast-enhanced thoracoabdominal CT, which showed a prostatic heteroplasia and several bone metastases at the pelvis and dorsolumbar vertebrae.

Cerebrospinal fluid (CSF) showed a normal cell count and elevated protein levels ( $83 \mathrm{mg} / \mathrm{dL})$ and CSF/serum albumin ratio (19.4). A serologic panel of onconeural antibodies inclusive of anti-Hu and anti-CV2 was negative, while antigAchR was positive at low titer $(0.05 \mathrm{nmol} / \mathrm{L})$. The patient was treated with intravenous steroid pulses (methylprednisolone $1 \mathrm{~g} /$ day for 5 days), then tapered to oral prednisone $50 \mathrm{mg} /$ day. This led to prompt resolution of the orthostatic hypotension (blood pressure of 130/80 $\mathrm{mmHg}$, unchanged in supine and standing position), in addition to maintenance of regular bowel movements after colonoscopic decompression. It was not possible to wean off the urinary catheter, possibly as a consequence of the prostate cancer. During hospitalization, he developed severe coronavirus disease 2019 and prednisone was discontinued, leading to relapse of ACPO. The patient was eventually commenced on hormone therapy for prostate cancer and transferred to a long-term care facility. 


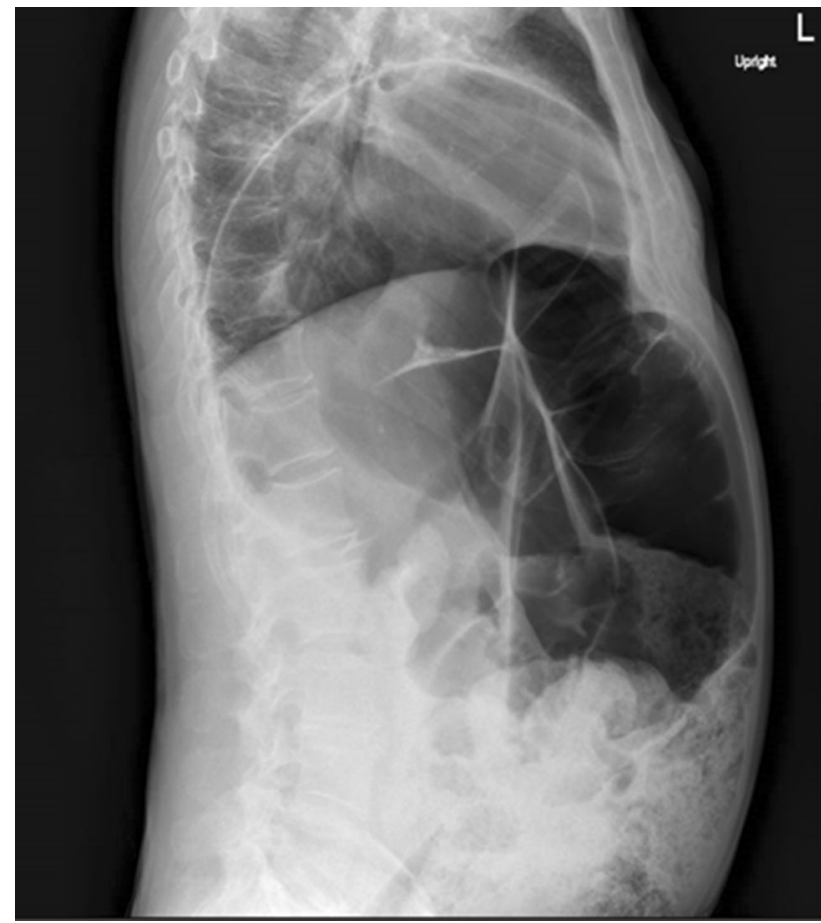

Fig. 1 Abdominal X-ray showing a massive colonic dilatation, consistent with acute colonic pseudo-obstruction

To our knowledge, this is the first report of subacuteonset PAN associated with prostate cancer. The patient at issue developed diffuse autonomic failure involving the sympathetic, parasympathetic and enteric nervous system (causing orthostatic hypotension, acute urinary retention and ACPO, respectively), partially responsive to high-dose steroid therapy. This presentation is clinically indistinguishable from AAG. Screening for occult malignancy by $\mathrm{CT}$ revealed a prostatic heteroplasia with bone secondaries, otherwise asymptomatic.

Prostate cancer is the second most frequent cancer diagnosis made in men and the fifth leading cause of death worldwide [5]. It has been rarely reported in association with paraneoplastic neurological syndromes (PNS): in the most recent review on this topic, among 37 reported cases with PNS and prostate cancer, only one patient presented with anti-Hu-associated limited gastrointestinal neuropathy, and no one with pandysautonomia [6].

Anti-gAchR antibodies at intermediate or low titers have been found in three patients with prostate cancer (in two cases with values in the range of $0.10-0.99 \mathrm{nmol} / \mathrm{L}$, in one case with values in the range $0.03-0.09 \mathrm{nmol} / \mathrm{L}$ ) in a single study describing the frequency of anti-gAchR seropositivity among 15,000 patients evaluated for paraneoplastic antibodies [4]. However, no detailed clinical information is available for these specific subjects.

Our patient was found to have low levels of anti-gAchR antibodies, which have been demonstrated to be quite nonspecific for pandysautonomia at this titer [1]. Therefore, it is not clear whether these antibodies had a pathogenic role in determining PAN in our patient, or represent an epiphenomenon possibly related to the presence of prostate cancer. This notwithstanding, the marked improvement of autonomic symptoms following steroid therapy suggests that PAN was immune-mediated. In general, treatment of PAN is first directed at eradicating the underlying malignancy, but immunotherapy may also be attempted to control dysautonomic symptoms [1]. While plasma exchange and intravenous immunoglobulin might be beneficial in anti-gAchR-mediated PAN, high-dose corticosteroids might be more effective in PAN forms related to onconeural antibodies.

In conclusion, subacute diffuse autonomic failure should be considered among the spectrum of PNS associated with prostate cancer. As in our patient, PAN may precede the diagnosis of cancer. Therefore, prompt screening for occult malignancies should be performed in adult patients presenting with subacute pandysautonomia, as this may have an impact on cancer prognosis.

Author contributions LM drafted the manuscript; LM and MN contributed to the acquisition of data; all authors contributed to the analysis of data; MN, PC and MG critically revised the manuscript for intellectual content.

\section{Compliance with ethical standards}

Conflict of interest The authors report no relevant disclosures, conflicts of interest or funding sources for this work.

Ethical approval We confirm that approval of an institutional review board was not required for this work. We confirm that we have read the Journal's position on issues involved in ethical publication and affirm that this work is consistent with those guidelines.

\section{References}

1. Golden EP, Vernino S (2019) Autoimmune autonomic neuropathies and ganglionopathies: epidemiology, pathophysiology, and therapeutic advances. Clin Auton Res 29:277-288

2. Lucchinetti CF, Kimmel DW, Lennon VA (1998) Paraneoplastic and oncologic profiles of patients seropositive for type 1 antineuronal nuclear autoantibodies. Neurology 50(3):625-657

3. Yu Z, Kryzer TJ, Griesmann GE, Kim K, Benarroch EE, Lennon VA (2001) CRMP-5 neuronal autoantibody: marker of lung cancer and thymoma-related autoimmunity. Ann Neurol 49(2):146-154

4. McKeon A, Lennon VA, Lachance DH, Fealey RD, Pittock SJ (2009) Ganglionic acetylcholine receptor autoantibody: oncological, neurological, and serological accompaniments. Arch Neurol 66(6):735-741

5. Rawla P (2019) Epidemiology of prostate cancer. World J Oncol 10(2):63-89

6. Storstein A, Raspotnig M, Vitaliani R, Giometto B, Graus F, Grisold W, Honnorat J, Vedeler CA (2016) Prostate cancer, Hu antibodies and paraneoplastic neurological syndromes. J Neurol 263(5):1001-1007 